University of Windsor

Scholarship at UWindsor

Summer 2009

\title{
An approach to multiattribute decision making with interval- valued intuitionistic fuzzy assessments and incomplete weights
}

Zhou-Jing Wang

Kevin W. Li Dr.

University of Windsor

Weize Wang

Follow this and additional works at: https://scholar.uwindsor.ca/odettepub

Part of the Business Commons

\section{Recommended Citation}

Wang, Zhou-Jing; Li, Kevin W. Dr.; and Wang, Weize. (2009). An approach to multiattribute decision making with interval-valued intuitionistic fuzzy assessments and incomplete weights. Information Sciences, 179 (17), 3026-3040.

https://scholar.uwindsor.ca/odettepub/65

This Article is brought to you for free and open access by the Odette School of Business at Scholarship at UWindsor. It has been accepted for inclusion in Odette School of Business Publications by an authorized administrator of Scholarship at UWindsor. For more information, please contact scholarship@uwindsor.ca. 
An Approach to Multiattribute Decision Making with Interval-Valued Intuitionistic Fuzzy Assessments and Incomplete Weights

$5 \quad{ }^{b}$ Odette School of Business, University of Windsor, Windsor, Ontario N9B 3P4, Canada

\section{Abstract}

8 This article proposes an approach to multiattribute decision making with incomplete 9 attribute weight information where individual assessments are provided as interval-valued

10 intuitionistic fuzzy numbers (IVIFNs). By employing a series of optimization models, the 11 proposed approach derives a linear program for determining attribute weights. The 12 weights are subsequently used to synthesize individual IVIFN assessments into an 13 aggregated IVIFN value for each alternative. In order to rank alternatives based on their 14 aggregated IVIFN values, a novel method is developed for comparing two IVIFNs by 15 introducing two new functions: the membership uncertainty index and the hesitation 16 uncertainty index. An illustrative investment decision problem is employed to 17 demonstrate how to apply the proposed procedure and comparative studies are conducted 18 to show its overall consistency with existing approaches.

19 Keywords: Multiattribute decision making, interval-valued intuitionistic fuzzy numbers 20 (IVIFNs), uncertainty index, linear programming

\section{1. Introduction}

22 Since the seminal work of Zadeh [39], the traditional 0-1 logic has been extended to 23 fuzzy logic, characterized by a membership function between 0 and 1 . This extension has 24 triggered significant theoretical developments and numerous successful industrial 25 applications [17, 41], and provides a powerful alternative other than probability theory to 26 characterize uncertainty, imprecision, and vagueness in many fields [40]. Intuitionistic 27 fuzzy sets (IFSs), initiated by Atanassov [1], represent one of the key theoretical 28 developments, which considers not only to what degree an element belongs to a particular

\footnotetext{
* Corresponding author. Telephone: +1 5192533000 ext. 3456; fax: +1 5199737073.

Email: kwli@uwindsor.ca (K.W.Li), wangzj@xmu.edu.cn (Z. Wang).
} 
29 set (membership function) but also to what degree this element does not belong to the set

30 (nonmembership function). The notion of IFSs is further generalized [3] by allowing the

31 membership and nonmembership functions to assume interval values, thereby introducing

32 the concept of interval-valued intuitionistic fuzzy sets (IVIFSs).

33 From a voting perspective, the membership function of an IFS can be loosely 34 regarded as the percentage of approval votes, the nonmembership function can be 35 interpreted as the rejection percentage, and the remaining portion that is not included in 36 either the membership or nonmembership function can be conveniently treated as 37 abstention. Due to these distinct features in characterizing vagueness and uncertainty in 38 human decision making processes, IFSs have been widely employed to develop diverse 39 decision aid tools. For instance, the concept of score functions is introduced by Chen and 40 Tan [6] to evaluate alternatives under multiple attributes where assessments of each 41 alternative against the attributes are expressed as vague values, or equivalently, 42 intuitionistic fuzzy numbers as pointed out by Deschrijver and Kerre [9]. Subsequently, 43 Hong and Choi [14] indicate that the score function cannot discriminate some alternatives 44 although they are apparently different and, hence, propose an accuracy function to 45 measure how accurate are the membership and nonmembership (or negation in the vague 46 set term) functions, thereby furnishing additional discrimination powers. Liu and Wang 47 [22] extend this research by first introducing an evaluation function based on $t$-norm and $48 \quad t$-conorm and, then defining an intuitionistic fuzzy point operator and developing several 49 new score functions based on the evaluation function and point operator. If a score 50 function is employed to rank alternatives, a higher score value means a more preferred 51 alternative.

52 Another active research topic is the investigation of multiattribute decision making by 53 introducing intuitionistic fuzzy aggregation operators. Xu and Yager [37] and Xu [32] 54 examine geometric and arithmetic aggregation operators, respectively. Multiattribute 55 decision making under IFSs is further investigated by $\mathrm{Li}$ [20], where a series of 56 optimization models are introduced and manipulated to generate optimal attribute 57 weights. The applications of IFSs are also extended to decision situations involving 58 multiple decision-makers (DMs): Szmidt and Kacprzyk [27] put forward some solution 59 concepts in group decision making with intuitionistic fuzzy preference relations, and 
60 Szmidt and Kacprzyk [28] further investigate how to reach consensus with intuitionistic 61 fuzzy preference relations. Atanassov et al. [4] also present an algorithm for multi-person 62 multiattribute decision making with crisp weights and intuitionistic fuzzy attribute values. $63 \mathrm{Xu}$ [33] defines consistent, incomplete, and acceptable preference relations and develops 64 another approach to group decision making under the intuitionistic fuzzy environment.

65 With the aforesaid extensive research on applying IFSs to decision analysis, it is 66 natural to expect that IVIFSs play a significant role in enriching decision modeling. 67 However, the extension from exact numbers to interval values for the membership and 68 nonmembership functions of IFSs poses considerable challenges in working with IVIFSs. 69 Current research mainly focuses on basic operations and relations of IVIFSs as well as 70 their properties [2]. Correlation and coefficient of correlation are first introduced by 71 Bustince and Burillo [5], and then generalized to a general probability space [13]. 72 Subsequently, Hung and Wu [15] develop a so-called "centroid" approach to calculating 73 the correlation coefficient of IVIFSs. Another method is proposed by Xu [31], which 74 possesses a key property that the correlation coefficient of two IVIFSs is one if and only 75 if the two IVIFSs are identical. Other aspects of IVIFSs are also investigated, such as 76 topological properties [25], relationships between IFSs, L-fuzzy sets, interval-valued 77 fuzzy sets and IVIFSs [7-9], and the entropy and subsethood of IVIFSs [23]. It is still at 78 an inceptive stage to apply IVIFSs to decision modeling and limited literature exists in 79 this specialized area. Xu [34] proposes some aggregation operators for interval-valued 80 intuitionistic fuzzy information and applies them to multiattribute decision analysis. Xu 81 and Yager [38] further investigate dynamic intuitionistic fuzzy aggregation operators and 82 devise two procedures for dynamic intuitionistic fuzzy multiattribute decision making 83 with intuitionistic fuzzy numbers (IFNs) or interval-valued intuitionistic fuzzy numbers 84 (IVIFNs).

85 Multiattribute decision approaches provide decision aid by examining tradeoffs 86 among alternative performances over multiple attributes [16]. Key information required 87 in a multiattribute decision model includes attribute values or performance measures 88 (individual assessments on alternatives against each attribute), attribute weights 89 (reflecting the importance of each attribute to the overall decision problem), and a 90 mechanism to synthesize this information into an aggregated value or assessment for each 
91 alternative. With ever increasing complexity in many decision situations in reality, it is

92 often a challenge for a decision-maker (DM) to provide attribute values and weights in a

93 precise manner. Therefore, a general trend in the literature is to investigate decision

94 models with incomplete information. For instance, attribute values have been relaxed to

95 be a range rather than an exact value [4, 6, 14, 18, 20, 22, 27-30, 33-35, 38], and

96 incomplete attribute weight information has also been extensively investigated from

97 different perspectives [18, 26, 36]. In addition, more and more research along this

98 direction has been conducted within a fuzzy or intuitionistic fuzzy framework [14, 19-22,

99 27-30, 33-36, 38]. The purpose of this article is to propose a novel approach to

100 multiattribute decision analysis in which attribute values are expressed as IVIFNs and

101 incomplete attribute weights are identified as a set of linear constraints that may take any

102 form as those in [18, 26, 36]. To rank alternatives based on their aggregated IVIFN

103 values, a new method is devised to compare any two IVIFNs in Section 3. To obtain

104 aggregated IVIFN values, this approach, motivated by the treatments in [20], starts with

105 manipulating a series of linear and nonlinear programming models, and eventually

106 derives a linear program to determine attribute weights for aggregating individual IVIFN

107 assessments into a single IVIFN value for each alternative (Section 4).

108 Intuitively, extending from IFNs to IVIFNs furnishes additional capability to handle 109 vague information because the membership and nonmembership degrees are only needed

110 to be expressed as ranges of values rather than exact values. When the uncertainty in an

111 IVIFN's membership and nonmembership degrees diminishes to zero, the IVIFN is

112 reduced to an IFN. Therefore, compared to the multiattribute decision models in existing

113 literature [14, 20, 22, 36, 38], the proposed approach makes a useful contribution by

114 empowering a DM with more flexibility in tackling vagueness and uncertainty in its

115 assessments, thereby providing an effective means to applying IVIFNs in multiattribute

116 decision making with incomplete weights. Another key contribution of this article is the

117 novel comparison method for IVIFNs in Section 3, which is able to differentiate any two 118 IVIFNs.

119 An earlier version of this paper was presented at a conference and published in the 120 proceedings [30]. The current manuscript significantly expands the conference paper by 121 providing new theorems (Section 4) to validate the proposed approach and introducing a 
122 new method (Section 3) to compare two IVIFNs rather than depending on a TOPSIS

123 (technique for order performance by similarity to ideal solution [16]) based approach to

124 ranking alternatives. Moreover, this paper has been thoroughly rewritten to explain the

125 procedure more carefully and enhance its readability. The updated illustrative example in

126 Section 5 demonstrates that two alternatives cannot be distinguished by using the

127 TOPSIS approach in the conference paper, but a full ranking can be obtained by using the

128 newly designed approach to comparing two IVIFNs in Section 3. The approach here also

129 significantly differs from that reported in Wang and Wang [29], from the process of 130 determining attribute weights (eigenalue-based), to the aggregation operator (weighted 131 arithmetic average) and ranking method (only score and accuracy functions are employed 132 there).

133 The remainder of this paper is organized as follows: Section 2 reviews some basic 134 concepts related to IFSs and IVIFSs. A novel method is introduced for comparing any 135 two IVIFNs in Section 3. Section 4 establishes a linear programming approach to 136 multiattribute decision making under interval-valued intuitionistic fuzzy environment. A 137 numerical example is developed to demonstrate how to apply the proposed approach and 138 some comparative studies are conducted in Section 5, followed by some concluding 139 remarks in Section 6.

\section{2. Preliminaries}

141 Some basic concepts on IFSs and IVIFSs are introduced below to facilitate future 142 discussions.

143 Definition 2.1 (Atanassov [1]). Let a set $X$ be fixed, an intuitionistic fuzzy set (IFS) $A$ 144 in $X$ is defined as

$145 \quad A=\left\{<x, \mu_{A}(x), v_{A}(x)>\mid x \in X\right\}$

146 where the functions $\mu_{A}: X \rightarrow[0,1], x \in X, \mu_{A}(x) \in[0,1]$ and $v_{A}: X \rightarrow[0,1], x \in X$, $147 v_{A}(x) \in[0,1]$ satisfy the condition $0 \leq \mu_{A}(x)+v_{A}(x) \leq 1, \forall x \in X$.

$148 \mu_{A}(x)$ and $v_{A}(x)$ denote the degrees of membership and nonmembership of element $149 x \in X$ to set $A$, respectively. $\pi_{A}(x)=1-\mu_{A}(x)-v_{A}(x)$ is usually called the intuitionistic 150 fuzzy index of $x \in A$, representing the degree of indeterminacy or hesitation of $x$ to $A$. It is 
obvious that $0 \leq \pi_{A}(x) \leq 1$ for every $x \in X$.

Deschrijver and Kerre [9] have shown that IFSs are equivalent to interval-valued fuzzy sets (also called vague sets [10]) and both can be regarded as L-fuzzy sets in the sense of Goguen [11].

In reality, it may not be easy to identify exact values for the membership and nonmembership degrees of an element to a set. In this case, a range of values may be a more appropriate measurement to accommodate the vagueness. As such, Atanassov and Gargov [3] introduce the notion of interval-valued intuitionistic fuzzy set (IVIFS).

Definition 2.2 (Atanassov and Gargov [3]). Let $X$ be a non-empty set of the universe, and $D[0,1]$ be the set of all closed subintervals of $[0,1]$, an interval-valued intuitionistic fuzzy set (IVIFS) $\tilde{A}$ in $X$ is defined by

$$
\tilde{A}=\left\{<x, \tilde{\mu}_{\tilde{A}}(x), \tilde{v}_{\tilde{A}}(x)>\mid x \in X\right\}
$$

where $\tilde{\mu}_{\tilde{A}}: X \rightarrow D[0,1], \quad \tilde{v}_{\tilde{A}}: X \rightarrow D[0,1]$, with the condition $0 \leq \sup \left(\tilde{\mu}_{\tilde{A}}(x)\right)+$ $\sup \left(\tilde{v}_{\tilde{A}}(x)\right) \leq 1$ for any $x \in X$.

Similarly, the intervals $\tilde{\mu}_{\tilde{A}}(x)$ and $\tilde{v}_{\tilde{A}}(x)$ denote the degree of membership and nonmembership of $x$ to $A$, respectively. But, here, for each $x \in X, \tilde{\mu}_{\tilde{A}}(x)$ and $\tilde{v}_{\tilde{A}}(x)$ are closed intervals rather than real numbers and their lower and upper boundaries are denoted by $\tilde{\mu}_{\tilde{A}}^{L}(x), \tilde{\mu}_{\tilde{A}}^{U}(x), \tilde{v}_{\tilde{A}}^{L}(x), \tilde{v}_{\tilde{A}}^{U}(x)$, respectively. Therefore, another equivalent way to express an IVIFS $\tilde{A}$ is

$$
\tilde{A}=\left\{<x,\left[\tilde{\mu}_{\tilde{A}}^{L}(x), \tilde{\mu}_{\tilde{A}}^{U}(x)\right],\left[\tilde{v}_{\tilde{A}}^{L}(x), \tilde{V}_{\tilde{A}}^{U}(x)\right]>\mid x \in X\right\},
$$

where $\tilde{\mu}_{\tilde{A}}^{U}(x)+\tilde{v}_{\tilde{A}}^{U}(x) \leq 1,0 \leq \tilde{\mu}_{\tilde{A}}^{L}(x) \leq \tilde{\mu}_{\tilde{A}}^{U}(x) \leq 1,0 \leq \tilde{v}_{\tilde{A}}^{L}(x) \leq \tilde{v}_{\tilde{A}}^{U}(x) \leq 1$.

Similar to IFSs, for each element $x \in X$ we can compute its hesitation interval relative to $\tilde{A}$ as:

$$
\tilde{\pi}_{\tilde{A}}(x)=\left[\tilde{\pi}_{\tilde{A}}^{L}(x), \tilde{\pi}_{\tilde{A}}^{U}(x)\right]=\left[1-\tilde{\mu}_{\tilde{A}}^{U}(x)-\tilde{v}_{\tilde{A}}^{U}(x), 1-\tilde{\mu}_{\tilde{A}}^{L}(x)-\tilde{v}_{\tilde{A}}^{L}(x)\right]
$$

If each of the intervals $\tilde{\mu}_{\tilde{A}}(x)$ and $\tilde{v}_{\tilde{A}}(x)$ contains only one real value, i.e., if for every $x \in X$,

$$
\mu_{\tilde{A}}(x)=\tilde{\mu}_{\tilde{A}}^{L}(x)=\tilde{\mu}_{\tilde{A}}^{U}(x), v_{\tilde{A}}(x)=\tilde{v}_{\tilde{A}}^{L}(x)=\tilde{v}_{\tilde{A}}^{U}(x)
$$


then, the given IVIFS $\tilde{A}$ is degraded to an ordinary IFS.

179 For any given $x$, the pair $\left(\tilde{\mu}_{\tilde{A}}(x), \tilde{v}_{\tilde{A}}(x)\right)$ is called an interval-valued intuitionistic

180 fuzzy number (IVIFN) [34,38]. For convenience, the pair $\left(\tilde{\mu}_{\tilde{A}}(x), \tilde{v}_{\tilde{A}}(x)\right)$ is often denoted

181 by $([a, b],[c, d])$, where $[a, b] \in D[0,1],[c, d] \in D[0,1]$ and $b+d \leq 1$.

\section{Remark 2.1}

183 For IFSs, $\pi_{A}(x)=1-\mu_{A}(x)-v_{A}(x)$ measures a DM's hesitation about the membership 184 of $x$ to $A$ and also represents the DM's uncertainty. For IVIFSs, the uncertainty comes 185 from three sources: membership uncertainty in $\left[\tilde{\mu}_{\tilde{A}}^{L}(x), \tilde{\mu}_{\tilde{A}}^{U}(x)\right]$, nonmembership 186 uncertainty in $\left[\tilde{v}_{\tilde{A}}^{L}(x), \tilde{v}_{\tilde{A}}^{U}(x)\right]$, and hesitation uncertainty in $\tilde{\pi}_{\tilde{A}}(x)=\left[\tilde{\pi}_{\tilde{A}}^{L}(x), \tilde{\pi}_{\tilde{A}}^{U}(x)\right]=$ $\left[1-\tilde{\mu}_{\tilde{A}}^{U}(x)-\tilde{v}_{\tilde{A}}^{U}(x), 1-\tilde{\mu}_{\tilde{A}}^{L}(x)-\tilde{v}_{\tilde{A}}^{L}(x)\right]$. This differentiation of uncertainty sources plays an

188 instrumental role in devising a novel method for comparing two IVIFNs in Section 3.

\section{A novel method for comparing two IVIFNs}

In the proposed multiattribute decision approach in Section 4, the eventual evaluation of each alternative will be based on an aggregated IVIFN. In order to rank alternatives, it is necessary to consider how to compare two IVIFNs.

193 For intuitionistic fuzzy numbers (IFNs), Chen and Tan [6] introduce a score function, defined as the difference of membership and nonmembership function, to evaluate alternatives and, then, develop a multiattribute decision making approach under the IFS environment. Later, Hong and Choi [14] note that the score function alone cannot differentiate many IFNs even though they are obviously different. To make the comparison method more discriminatory, an accuracy function, defined as the sum of the membership and nonmembership function, is introduced to measure how accurate are the membership and nonmembership functions of an IFN. Subsequently, a procedure

202 decision making problems with IFNs [14]. Built upon the concepts of score and accuracy functions, Xu [32] devises a new approach to comparing two IFNs.

204 When the comparison of two IFNs is extended to the interval-valued case, a similar 205 line of thinking can be adopted. For instance, $\mathrm{Xu}$ [34] introduces the score and accuracy 206 functions for IVIFNs and applies them to compare two IVIFNs. However, due to the 
specific characteristics of intervals and the three different types of uncertainty (See

208 Remark 2.1), the score and accuracy functions together sometimes cannot tell the

209 difference between two IVIFNs. In this case, it is necessary to examine the difference

210 between two IVIFNs using two additional functions as detailed below. The first two

211 functions are proposed by $\mathrm{Xu}$ [34], but the last two are introduced in this research.

212 1. Score function: The difference between the membership and nonmembership 213 functions, $\tilde{\mu}_{\tilde{A}}=[a, b]$ and $\tilde{v}_{\tilde{A}}=[c, d]$. As these functions are interval-valued, the means

214 of the respective intervals are employed for the calculation. This difference is comparable 215 to the score function in the IFN case and, hence, we have:

216 Definition 3.1 (Xu [34]) For an IVIFN $\tilde{\alpha}=([a, b],[c, d])$, its score function is defined 217 as $S(\tilde{\alpha})=\frac{a+b-c-d}{2}$.

218 It is obvious that $-1 \leq S(\tilde{\alpha}) \leq 1$. The score function captures the overall degree of 219 belonging to a certain set by deducting its nonmembership from its membership function 220 and, hence, can be used as a basis to compare two IVIFNs. For two IVIFNs, the one with 221 a smaller score function corresponds to a smaller IVIFN. However, two different IVIFNs 222 may possess an identical score value as shown in the following example.

223 Example 3.1 Let $\tilde{\alpha}_{1}=([0.2,0.3],[0.2,0.3])$ and $\tilde{\alpha}_{2}=([0.4,0.5],[0.4,0.5])$. It is trivial 224 to confirm that $S\left(\tilde{\alpha}_{1}\right)=S\left(\tilde{\alpha}_{2}\right)=0$, but these two IVIFNs are obviously different.

2252 2. Accuracy function: When the score function alone cannot differentiate two 226 IVIFNs as shown in Example 3.1, additional information, the sum of the membership and 227 nonmembership functions, should now be considered. This idea is similar to the accuracy 228 function in [14] except that the mean values of the intervals are employed here.

229 Definition 3.2 (Xu [34]) For an IVIFN $\tilde{\alpha}=([a, b],[c, d])$, its accuracy function is 230 defined as $H(\tilde{\alpha})=\frac{a+b+c+d}{2}$.

231 Generally speaking, the accuracy function measures the amount of information 232 captured by the membership and nonmembership functions, and the remaining portion 233 characterizes the degree of hesitation. When the score function is the same for two 234 IVIFNs, the smaller the accuracy function, the larger the hesitation and, hence, the 
smaller the corresponding IVIFN. For the two IVIFNs in Example 3.1, since their score function value is identical but $H\left(\tilde{\alpha}_{1}\right)=0.5<H\left(\tilde{\alpha}_{2}\right)=0.9$, we have $\tilde{\alpha}_{1}<\tilde{\alpha}_{2}$.

It is clear that the introduction of the accuracy function increases the discriminatory power. Nevertheless, in some situations, the score and accuracy functions together still cannot tell the difference between two distinct IVIFNs. For instance,

Example 3.2 Let $\tilde{\alpha}_{1}=([0,0.4],[0.3,0.4]), \tilde{\alpha}_{2}=([0.1,0.3],[0.3,0.4]), \quad \tilde{\alpha}_{3}=([0,0.4]$, $[0.18,0.52]), \tilde{\alpha}_{4}=([0.05,0.35],[0.2,0.5]), \quad \tilde{\alpha}_{5}=([0.2,0.2],[0.3,0.4])$. It is easy to verify that $S\left(\tilde{\alpha}_{1}\right)=S\left(\tilde{\alpha}_{2}\right)=S\left(\tilde{\alpha}_{3}\right)=S\left(\tilde{\alpha}_{4}\right)=S\left(\tilde{\alpha}_{5}\right)=-0.15 \quad$ and $\quad H\left(\tilde{\alpha}_{1}\right)=H\left(\tilde{\alpha}_{2}\right)=H\left(\tilde{\alpha}_{3}\right)=$

$243 H\left(\tilde{\alpha}_{4}\right)=H\left(\tilde{\alpha}_{5}\right)=0.55$. Therefore, these five IVIFNs are still indistinguishable.

244 As a matter of fact, for any two IVIFNs, as long as the means of their membership and nonmembership intervals are respectively equal, the score and accuracy functions of the two IVIFNs will be identical and, hence, indistinguishable under these two functions.

3. Membership uncertainty index function: When both score and accuracy functions fail to distinguish two IVIFNs, the difference of the uncertainty in the membership and nonmembership functions is considered.

Intuitively, the uncertainty of a membership (nonmembership) function is measured by the width of the interval: the wider a membership (nonmembership) interval, the more uncertain an element's membership (nonmembership) is. When the width of the interval diminishes to zero, it is known exactly to what degree an element belongs (does not belong) to a particular set. In this case, no uncertainty exists about an element's membership (nonmembership) to the set.

Definition 3.3 For an IVIFN $\tilde{\alpha}=([a, b],[c, d])$, its membership uncertainty index is defined as $T(\tilde{\alpha})=b+c-a-d$.

258 It is easy to tell that $-1 \leq T(\tilde{\alpha}) \leq 1$. Understandably, when the score and accuracy 259 functions are equal for two IVIFNs, the larger a $T(\cdot)$ value, the smaller the corresponding 260 IVIFN is. For the five IVIFNs in Example 3.2, applying Definition 3.3 yields $T\left(\tilde{\alpha}_{1}\right)=0.3$, $261 T\left(\tilde{\alpha}_{2}\right)=0.1, T\left(\tilde{\alpha}_{3}\right)=0.06, T\left(\tilde{\alpha}_{4}\right)=0$, and $T\left(\tilde{\alpha}_{5}\right)=-0.1$. As $T\left(\tilde{\alpha}_{1}\right)>T\left(\tilde{\alpha}_{2}\right)>T\left(\tilde{\alpha}_{3}\right)>$ $262 T\left(\tilde{\alpha}_{4}\right)>T\left(\tilde{\alpha}_{5}\right)$, one can have $\tilde{\alpha}_{1}<\tilde{\alpha}_{2}<\tilde{\alpha}_{3}<\tilde{\alpha}_{4}<\tilde{\alpha}_{5}$.

263 However, with the three functions, $S(\cdot), H(\cdot)$, and $T(\cdot)$, some IVIFNs still cannot be 
264

265

266

267

268

269

270

271

272

273

274

275

276

277

278

279

280

281

282

283

284

285

286

287

288

289

290

differentiated. For example,

Example 3.3 Assume that $\tilde{\alpha}_{1}=([0.05,0.35],[0.25,0.55]), \tilde{\alpha}_{2}=([0.1,0.3],[0.3,0.5])$,

$$
\tilde{\alpha}_{3}=([0.15,0.25],[0.35,0.45]), \quad \tilde{\alpha}_{4}=([0.2,0.2],[0.4,0.4]) \text {, then, }
$$

$$
S\left(\tilde{\alpha}_{1}\right)=S\left(\tilde{\alpha}_{2}\right)=S\left(\tilde{\alpha}_{3}\right)=S\left(\tilde{\alpha}_{4}\right)=-0.2
$$$$
H\left(\tilde{\alpha}_{1}\right)=H\left(\tilde{\alpha}_{2}\right)=H\left(\tilde{\alpha}_{3}\right)=H\left(\tilde{\alpha}_{4}\right)=0.6
$$$$
T\left(\tilde{\alpha}_{1}\right)=T\left(\tilde{\alpha}_{2}\right)=T\left(\tilde{\alpha}_{3}\right)=T\left(\tilde{\alpha}_{4}\right)=0
$$

Therefore, $\tilde{\alpha}_{1}, \tilde{\alpha}_{2}, \tilde{\alpha}_{3}$, and $\tilde{\alpha}_{4}$ cannot be differentiated by using $S(\cdot), H(\cdot)$, and $T(\cdot)$.

In general, for any two IVIFNs $\tilde{\alpha}=\left(\left[a_{1}, b_{1}\right],\left[c_{1}, d_{1}\right]\right)$ and $\tilde{\beta}=\left(\left[a_{2}, b_{2}\right],\left[c_{2}, d_{2}\right]\right)$, if $a_{1}+$ $b_{1}=a_{2}+b_{2}, c_{1}+d_{1}=c_{2}+d_{2}, b_{1}+c_{1}=b_{2}+c_{2}$, and $a_{1}+d_{1}=a_{2}+d_{2}$, then, $S(\tilde{\alpha})=S(\tilde{\beta})$, $H(\tilde{\alpha})=H(\tilde{\beta})$, and $T(\tilde{\alpha})=T(\tilde{\beta})$, hence, the three functions will not be able to distinguish these two IVIFNs. In this case, the uncertainty contained in the hesitation interval has to be examined.

4. Hesitation uncertainty index function: Once again, the uncertainty in the hesitation interval, $\tilde{\pi}_{\tilde{A}}(x)=\left[\tilde{\pi}_{\tilde{A}}^{L}(x), \tilde{\pi}_{\tilde{A}}^{U}(x)\right]=[1-b-d, 1-a-c]$, is measured by its width.

Definition 3.4 For an IVIFN $\tilde{\alpha}=([a, b],[c, d])$, its hesitation uncertainty index is defined as $G(\tilde{\alpha})=b+d-a-c$.

When the other three functions are equal, a larger hesitation uncertainty corresponds to a smaller IVIFN. By introducing $G(\cdot)$, the four IVIFNs in Example 3.3 can be ranked. As $G\left(\tilde{\alpha}_{1}\right)=0.6>G\left(\tilde{\alpha}_{2}\right)=0.4>G\left(\tilde{\alpha}_{3}\right)=0.2>G\left(\tilde{\alpha}_{4}\right)=0, \tilde{\alpha}_{1}<\tilde{\alpha}_{2}<\tilde{\alpha}_{3}<\tilde{\alpha}_{4}$.

Given these analyses, we can now introduce a procedure to compare any two IVIFSs. Definition 3.5 For any two IVIFNs $\tilde{\alpha}=\left(\left[a_{1}, b_{1}\right],\left[c_{1}, d_{1}\right]\right)$ and $\tilde{\beta}=\left(\left[a_{2}, b_{2}\right],\left[c_{2}, d_{2}\right]\right)$,

If $S(\tilde{\alpha})<S(\tilde{\beta})$, then $\tilde{\alpha}$ is smaller than $\tilde{\beta}$, denoted by $\tilde{\alpha}<\tilde{\beta}$;

If $S \tilde{(\alpha)}>S(\tilde{\beta})$, then $\tilde{\alpha}$ is greater than $\tilde{\beta}$, denoted by $\tilde{\alpha}>\tilde{\beta}$;

If $S(\tilde{\alpha})=S(\tilde{\beta})$, then

1) If $H(\tilde{\alpha})<H(\tilde{\beta})$, then $\tilde{\alpha}$ is smaller than $\tilde{\beta}$, denoted by $\tilde{\alpha}<\tilde{\beta}$;

2) If $H(\tilde{\alpha})>H(\tilde{\beta})$, then $\tilde{\alpha}$ is greater than $\tilde{\beta}$, denoted by $\tilde{\alpha}>\tilde{\beta}$;

3) If $H(\tilde{\alpha})=H(\tilde{\beta})$, then 
i) If $T(\tilde{\alpha})>T(\tilde{\beta})$, then $\tilde{\alpha}$ is smaller than $\tilde{\beta}$, denoted by $\tilde{\alpha}<\tilde{\beta}$;

ii) If $T(\tilde{\alpha})<T(\tilde{\beta})$, then $\tilde{\alpha}$ is greater than $\tilde{\beta}$, denoted by $\tilde{\alpha}>\tilde{\beta}$;

iii) If $T(\tilde{\alpha})=T(\tilde{\beta})$, then

\section{Remark 3.1}

a) If $G(\tilde{\alpha})>G(\tilde{\beta})$, then $\tilde{\alpha}$ is smaller than $\tilde{\beta}$, denoted by $\tilde{\alpha}<\tilde{\beta}$;

b) If $G(\tilde{\alpha})<G(\tilde{\beta})$, then $\tilde{\alpha}$ is greater than $\tilde{\beta}$, denoted by $\tilde{\alpha}>\tilde{\beta}$;

c) If $G(\tilde{\alpha})=G(\tilde{\beta})$, then $\tilde{\alpha}$ and $\tilde{\beta}$ represent the same information, denoted by $\tilde{\alpha}=\tilde{\beta}$

Definition 3.5 establishes a novel approach to comparing any two IVIFNs by taking a prioritized sequence of score, accuracy, membership uncertainty index, and hesitation uncertainty index functions. When two IVIFNs are compared, this sequence follows a logic order of examining the overall belonging degree, the level of accuracy or hesitation, the membership uncertainty index, and the hesitation uncertainty index. The comparison process continues until the two IVIFNs are distinguished by one of the four functions in Definition 3.5. Once these two IVIFNs are differentiated at a certain priority level, the calculation terminates and functions at lower priority levels will not be computed. This prioritized sequence of comparison method has many applications in reality. For instance, many Canadian research-intensive institutions recruit their tenure-track faculty members following a priority order of research first, teaching second, and service last. Theorem 3.1 below confirms that any two different IVIFNs will always be distinguishable by Definition 3.5.

Theorem 3.1 Let $\tilde{\alpha}=\left(\left[a_{1}, b_{1}\right],\left[c_{1}, d_{1}\right]\right)$ and $\tilde{\beta}=\left(\left[a_{2}, b_{2}\right],\left[c_{2}, d_{2}\right]\right)$ be two IVIFNs, then $\tilde{\alpha}=\tilde{\beta}$ iff $a_{1}=a_{2}, b_{1}=b_{2}, c_{1}=c_{2}, d_{1}=d_{2}$.

Proof: The sufficient condition obviously holds true. Next, if $\tilde{\alpha}=\tilde{\beta}$, then Definition 3.5 implies that $S(\tilde{\alpha})=S(\tilde{\beta}), \quad H(\tilde{\alpha})=H(\tilde{\beta}), \quad T(\tilde{\alpha})=T(\tilde{\beta})$, and $G(\tilde{\alpha})=G(\tilde{\beta})$. From the definitions of $S(\cdot), H(\cdot), T(\cdot)$, and $G(\cdot)$, we have

$$
\begin{aligned}
& a_{1}+b_{1}-c_{1}-d_{1}=a_{2}+b_{2}-c_{2}-d_{2}, a_{1}+b_{1}+c_{1}+d_{1}=a_{2}+b_{2}+c_{2}+d_{2} \\
& b_{1}+c_{1}-a_{1}-d_{1}=b_{2}+c_{2}-a_{2}-d_{2}, b_{1}+d_{1}-a_{1}-c_{1}=b_{2}+d_{2}-a_{2}-c_{2}
\end{aligned}
$$

By solving the four equations, we have $a_{1}=a_{2}, b_{1}=b_{2}, c_{1}=c_{2}, d_{1}=d_{2}$.

Q.E.D. 

in $D[0,1]$ is defined as: $\left[a_{1}, b_{1}\right] \leq\left[a_{2}, b_{2}\right]$ iff $a_{1} \leq a_{2}$ and $b_{1} \leq b_{2}$.

322 This definition can be treated as a special case of Definition 2.1 in [8] and, hence, $323<D[0,1], " \leq ">$ constitutes a complete lattice.

$324 \quad$ For any two IVIFNs, $\tilde{\alpha}$ and $\tilde{\beta}$, denote $\tilde{\alpha} \leq \tilde{\beta}$ iff $\tilde{\alpha}<\tilde{\beta}$ or $\tilde{\alpha}=\tilde{\beta}$.

325 Theorem 3.2 Let $\tilde{\alpha}=\left(\left[a_{1}, b_{1}\right],\left[c_{1}, d_{1}\right]\right)$ and $\tilde{\beta}=\left(\left[a_{2}, b_{2}\right],\left[c_{2}, d_{2}\right]\right)$ be two IVIFNs, if $326\left[a_{1}, b_{1}\right] \leq\left[a_{2}, b_{2}\right]$ and $\left[c_{1}, d_{1}\right] \geq\left[c_{2}, d_{2}\right]$, then $\tilde{\alpha} \leq \tilde{\beta}$.

327 Proof: Since $\left[a_{1}, b_{1}\right] \leq\left[a_{2}, b_{2}\right]$ and $\left[c_{1}, d_{1}\right] \geq\left[c_{2}, d_{2}\right]$, Definition 3.6 implies that 328 $a_{1} \leq a_{2}, b_{1} \leq b_{2}, c_{1} \geq c_{2}$, and $d_{1} \geq d_{2}$.

329 By the definition of score functions, we have $S(\tilde{\alpha})-S(\tilde{\beta})=\left(a_{1}+b_{1}-c_{1}-d_{1}\right) / 2-$ 330 $\left(a_{2}+b_{2}-c_{2}-d_{2}\right) / 2=\left(a_{1}+b_{1}-a_{2}-b_{2}\right) / 2+\left(c_{2}+d_{2}-c_{1}-d_{1}\right) / 2 \leq 0$. Two cases have to 331 be considered:

1) if $S(\tilde{\alpha})-S(\tilde{\beta})<0$, then $\tilde{\alpha}<\tilde{\beta}$ as per Definition 3.5. Otherwise,

2) if $S(\tilde{\alpha})-S(\tilde{\beta})=0$ then

$$
a_{1}+b_{1}-c_{1}-d_{1}=a_{2}+b_{2}-c_{2}-d_{2}
$$

Rearranging the terms yields

a) if $H(\tilde{\alpha})-H(\tilde{\beta})<0$ then $\tilde{\alpha}<\tilde{\beta}$ by Definition 3.5. Otherwise,

b) if $H(\tilde{\alpha})-H(\tilde{\beta})=0$, i.e., $H(\tilde{\alpha})-H(\tilde{\beta})=\left(a_{1}-a_{2}\right)+\left(b_{1}-b_{2}\right)=0$, then

$$
a_{1}+b_{1}=a_{2}+b_{2}
$$

(3.4) - (3.1) leads to $c_{1}+d_{1}=c_{2}+d_{2}$. By rearranging these terms, we have

$$
a_{1}-a_{2}=b_{2}-b_{1}, \quad c_{1}-c_{2}=d_{2}-d_{1}
$$


As $a_{1}-a_{2} \leq 0$ and $b_{2}-b_{1} \geq 0$, the first equation in (3.5) implies that $a_{1}-a_{2}=b_{2}-b_{1}=$

0. Similarly, as $c_{1}-c_{2} \leq 0$ and $d_{2}-d_{1} \geq 0$, the second part of (3.5) yields

$348 c_{1}-c_{2}=d_{2}-d_{1}=0$. Therefore, we have $a_{1}=a_{2}, b_{1}=b_{2}, c_{1}=c_{2}$, and $d_{1}=d_{2}$ and, hence, $\tilde{\alpha}=\tilde{\beta}$.

can be differentiated by the score and accuracy functions.

352

353

354

355

356

357

358

359

360

361

362

363

364

365

366

367

368

369

370

371

372

\section{An approach to multiattribute decision making with interval-valued intuitionistic fuzzy assessments and incomplete weights}

This section puts forward a framework for multiattribute decision making with incomplete weight information, where assessments of alternatives against attributes are given as interval-valued intuitionistic fuzzy numbers and incomplete attribute weight information is provided by the DM as a set of linear constraints.

\subsection{Problem formulations}

Given an alternative set $X=\left\{x_{1}, x_{2}, \cdots, x_{n}\right\}$, consisting of $n$ non-inferior decision alternatives, and an attribute set $A=\left(a_{1}, a_{2}, \cdots a_{m}\right)$. Each alternative is assessed on each of the $m$ attributes and the assessment is expressed as an IVIFN, describing the satisfaction and dissatisfaction degree of the alternative to a fuzzy concept of "excellence" as per a particular attribute. The decision problem is to select a most preferred alternative from $X$ or obtain a ranking of all alternatives based on the overall assessments of all alternatives on the $m$ attributes.

More Specifically, let $\tilde{R}=\left(\tilde{r}_{i j}\right)_{n \times m}=\left(\left(\left[a_{i j}, b_{i j}\right],\left[c_{i j}, d_{i j}\right]\right)\right)_{n \times m}$ be the interval-valued intuitionistic fuzzy decision matrix, where $\left[a_{i j}, b_{i j}\right]$ and $\left[c_{i j}, d_{i j}\right]$ are the membership and nonmembership intervals of alternative $x_{i}$ on attribute $a_{j}$ as per a fuzzy concept "excellence" given by a decision-maker (DM), indicating to what degree $x_{i}$ satisfies and does not satisfy the "excellence” requirement as per $a_{j}$, respectively. By Definition 2.2, $\left[a_{i j}, b_{i j}\right] \in D[0,1],\left[c_{i j}, d_{i j}\right] \in D[0,1]$, and $b_{i j}+d_{i j} \leq 1, \quad i=1,2, \cdots, n, j=1,2, \cdots, m$. It is clear that the lowest satisfaction degree of $x_{i}$ with respect to $a_{j}$ is $\left[a_{i j}, b_{i j}\right]$, as given in the 
373 membership function, and the highest satisfaction degree of $x_{i}$ with respect to $a_{j}$ is

$374\left[1-d_{i j}, 1-c_{i j}\right]$, in the case that all hesitation is treated as membership or satisfaction.

375 In a multiattribute decision making problem, different weights on attributes reflect 376 their varying importance in choosing the optimal alternative. Let $w=\left(w_{1}, w_{2}, \cdots, w_{m}\right)^{T}$ be 377 the attribute weight vector, where $w_{j} \geq 0, j=1,2, \cdots, m$, and the weight vector is often

378 normalized to one, i.e. $\sum_{j=1}^{m} w_{j}=1$. In reality, due to the increasing complexity of many

379 practical decision situations, the DM may not be confident in providing exact values for 380 attribute weights. Instead, the DM may only possess partial knowledge about attribute 381 weights [18]. This phenomenon has triggered significant research on developing decision 382 models for handling incomplete attribute weights [18,26,36]. Generally speaking, the 383 incomplete attribute weight information can be expressed as the following relationships 384 among the weights:

385 1) A weak ranking: $\left\{w_{j_{1}} \geq w_{j_{2}}\right\}, j_{1} \neq j_{2}$;

2) A strict ranking: $\left\{w_{j_{1}}-w_{j_{2}} \geq \varepsilon_{j_{1} j_{2}}(>0)\right\}, j_{1} \neq j_{2}$;

3) A ranking with multiples: $\left\{w_{j_{1}} \geq \alpha_{j_{1} j_{2}} w_{j_{2}}\right\}, 0 \leq \alpha_{j_{1} j_{2}} \leq 1, j_{1} \neq j_{2}$;

4) An interval form: $\left\{\beta_{j} \leq w_{j} \leq \beta_{j}+\varepsilon_{j}\right\}, 0 \leq \beta_{j}<\beta_{j}+\varepsilon_{j} \leq 1$;

5) A ranking of differences: $\left\{w_{j_{1}}-w_{j_{2}} \geq w_{j_{3}}-w_{j_{4}}\right\}$, for $j_{1} \neq j_{2} \neq j_{3} \neq j_{4}$.

394 of alternative $x_{i}$ is aggregated by incorporating attribute weights, it is expected that the 395 optimal satisfaction degree should also satisfy this condition, i.e., $396\left[a_{i j}, b_{i j}\right] \leq\left[\xi_{i j}, \eta_{i j}\right] \leq\left[1-d_{i j}, 1-c_{i j}\right]$. According to Definition 3.6, $\xi_{i j}$ and $\eta_{i j}$ should satisfy $397 \quad a_{i j} \leq \xi_{i j} \leq 1-d_{i j}$ and $b_{i j} \leq \eta_{i j} \leq 1-c_{i j}$.

398 Notice that as $a_{i j} \leq b_{i j}$, $c_{i j} \leq d_{i j}$ and $b_{i j}+d_{i j} \leq 1$, we have $a_{i j} \leq b_{i j} \leq 1-d_{i j} \leq 1-c_{i j}$.

\section{$399 \quad 4.2$ An optimization model for deriving aggregated IVIFN values}


Assume that the satisfaction degree interval of alternative $x_{i}$ with respect to $a_{j}$ is

401 given as $\left[\xi_{i j}, \eta_{i j}\right]$, its aggregated interval value incorporating attribute weights can be

402 expressed as

403

$$
\left[z_{i}^{L}, z_{i}^{U}\right]=\left[\sum_{j=1}^{m} \xi_{i j} w_{j}, \sum_{j=1}^{m} \eta_{i j} w_{j}\right], i=1,2, \ldots, n .
$$

404 As the aggregated value $\left[z_{i}^{L}, z_{i}^{U}\right]$ reflects the overall satisfaction degree of alternative $405 x_{i}$ to the fuzzy concept of “excellence", the greater the $\left[z_{i}^{L}, z_{i}^{U}\right]$, the better the alternative $406 x_{i}$ is. Therefore, a reasonable attribute weight vector $\left(w_{1}, w_{2}, \cdots, w_{m}\right)^{T}$ is to maximize $407\left[z_{i}^{L}, z_{i}^{U}\right]$. Motivated by the optimization models for multiattribute decision making under 408 IFSs presented by Li [20], this article extends the idea and proposes a similar framework 409 to handle multiattribute decision making problems with incomplete attribute weights 410 under IVIFSs.

411 As per Definition 3.6, the following two optimization models can thus be established 412 for each alternative:

413

$$
\begin{aligned}
& \max \left\{z_{i}^{L}=\sum_{j=1}^{m} \xi_{i j} w_{j}\right\} \\
& \text { s.t. }\left\{\begin{array}{l}
a_{i j} \leq \xi_{i j} \leq 1-d_{i j} \quad(i=1,2, \cdots, n ; j=1,2, \cdots, m), \\
w \in H, \\
\sum_{j=1}^{m} w_{j}=1
\end{array}\right.
\end{aligned}
$$

414 and

415

$$
\begin{aligned}
& \max \left\{z_{i}^{U}=\sum_{j=1}^{m} \eta_{i j} w_{j}\right\} \\
& \text { s.t. }\left\{\begin{array}{l}
b_{i j} \leq \eta_{i j} \leq 1-c_{i j} \\
w \in H, \\
\sum_{j=1}^{m} w_{j}=1
\end{array} \quad(i=1,2, \cdots, n ; j=1,2, \cdots, m),\right.
\end{aligned}
$$

416 for each $i=1,2, \ldots, n$. 
417 Similar to the treatment in Li [20], (4.1) can be converted to the following two linear 418 programs:

419

$$
\min \left\{z_{i}^{L L}=\sum_{j=1}^{m} a_{i j} w_{j}\right\}
$$

$$
\text { s.t. }\left\{\begin{array}{c}
w \in H, \\
\sum_{j=1}^{m} w_{j}=1
\end{array}\right.
$$

420 and

$$
\max \left\{z_{i}^{L U}=\sum_{j=1}^{m}\left(1-d_{i j}\right) w_{j}\right\}
$$

421

$$
\text { s.t. }\left\{\begin{array}{c}
w \in H, \\
\sum_{j=1}^{m} w_{j}=1
\end{array}\right.
$$

422 for each $i=1,2, \ldots, n$.

423 By following the same manner, (4.2) is transformed to the following two linear 424 programs:

$$
\min \left\{z_{i}^{U L}=\sum_{j=1}^{m} b_{i j} w_{j}\right\}
$$

425

$$
\text { s.t. }\left\{\begin{array}{c}
w \in H, \\
\sum_{j=1}^{m} w_{j}=1
\end{array}\right.
$$

426 and

$$
\begin{aligned}
& \max \left\{z_{i}^{U U}=\sum_{j=1}^{m}\left(1-c_{i j}\right) w_{j}\right\} \\
& \text { s.t. }\left\{\begin{array}{l}
w \in H, \\
\sum_{j=1}^{m} w_{j}=1
\end{array}\right.
\end{aligned}
$$

428 for each $i=1,2, \ldots, n$.

429 Models (4.3)-(4.6) are standard linear programs that can be conveniently solved.

430 Denote their optimal solutions by $\tilde{W}_{i}^{L L}=\left(\tilde{w}_{i 1}^{L L}, \tilde{w}_{i 2}^{L L}, \cdots, \tilde{w}_{i m}^{L L}\right)^{T}, \tilde{W}_{i}^{L U}=\left(\tilde{w}_{i 1}^{L U}, \tilde{w}_{i 2}^{L U}, \cdots, \tilde{w}_{i m}^{L U}\right)^{T}$,

$431 \tilde{W}_{i}^{U L}=\left(\tilde{w}_{i 1}^{U L}, \tilde{w}_{i 2}^{U L}, \cdots, \tilde{w}_{i m}^{U L}\right)^{T}$ and $\tilde{W}_{i}^{U U}=\left(\tilde{w}_{i 1}^{U U}, \tilde{w}_{i 2}^{U U}, \cdots, \tilde{w}_{i m}^{U U}\right)^{T}(i=1,2, \ldots, n)$, respectively, 432 and let 


$$
\begin{aligned}
& \tilde{z}_{i}^{L L} \triangleq \sum_{j=1}^{m} a_{i j} \tilde{w}_{i j}^{L L} \\
& \tilde{z}_{i}^{U L} \triangleq \sum_{j=1}^{m} b_{i j} \tilde{w}_{i j}^{U L} \\
& \tilde{z}_{i}^{L U} \triangleq \sum_{j=1}^{m}\left(1-d_{i j}\right) \tilde{w}_{i j}^{L U} \\
& \tilde{z}_{i}^{U U} \triangleq \sum_{j=1}^{m}\left(1-c_{i j}\right) \tilde{w}_{i j}^{U U}
\end{aligned}
$$

434 for each $i=1,2, \ldots, n$. Then Theorem 4.1 follows.

435 Theorem 4.1 Assume that $\tilde{z}_{i}^{L L}, \tilde{z}_{i}^{U L}, \tilde{z}_{i}^{L U}$, and $\tilde{z}_{i}^{U U}$ are respectively defined by (4.7),

436 then $\tilde{z}_{i}^{L L} \leq \tilde{z}_{i}^{U L}, \tilde{z}_{i}^{L U} \leq \tilde{z}_{i}^{U U}$, and $\tilde{z}_{i}^{U L} \leq \tilde{z}_{i}^{L U}, i=1,2, \ldots, n$.

437 Proof. Note that $\tilde{W}_{i}^{L L}=\left(\tilde{w}_{i 1}^{L L}, \tilde{w}_{i 2}^{L L}, \cdots, \tilde{w}_{i m}^{L L}\right)^{T}, \tilde{W}_{i}^{L U}=\left(\tilde{w}_{i 1}^{L U}, \tilde{w}_{i 2}^{L U}, \cdots, \tilde{w}_{i m}^{L U}\right)^{T}, \quad \tilde{W}_{i}^{U L}=\left(\tilde{w}_{i 1}^{U L}\right.$,

$\left.438 \tilde{w}_{i 2}^{U L}, \cdots, \tilde{w}_{i m}^{U L}\right)^{T}$, and $\tilde{W}_{i}^{U U}=\left(\tilde{w}_{i 1}^{U U}, \tilde{w}_{i 2}^{U U}, \cdots, \tilde{w}_{i m}^{U U}\right)^{T}$ are optimal solutions of (4.3), (4.4), (4.5),

439 and (4.6), respectively, and $a_{i j} \leq b_{i j}$ and $c_{i j} \leq d_{i j}$. According to (4.3), we have

$$
\tilde{z}_{i}^{L L} \triangleq \sum_{j=1}^{m} a_{i j} \tilde{w}_{i j}^{L L} \leq \sum_{j=1}^{m} a_{i j} \tilde{w}_{i j}^{U L} \leq \sum_{j=1}^{m} b_{i j} \tilde{w}_{i j}^{U L} \triangleq \tilde{z}_{i}^{U L}
$$

441 where the first inequality is due to the fact that $\tilde{w}_{i j}^{L L}$ is an optimal solution of (4.3) and $442 \tilde{w}_{i j}^{U L}$ is a feasible solution of this minimization problem, and the second inequality holds 443 true as $a_{i j} \leq b_{i j}$.

$444 \quad$ Similarly, from (4.6), one can obtain

$$
\tilde{z}_{i}^{L U} \triangleq \sum_{j=1}^{m}\left(1-d_{i j}\right) \tilde{w}_{i j}^{L U} \leq \sum_{j=1}^{m}\left(1-c_{i j}\right) \tilde{w}_{i j}^{L U} \leq \sum_{j=1}^{m}\left(1-c_{i j}\right) \tilde{w}_{i j}^{U U} \triangleq \tilde{z}_{i}^{U U}
$$

446 where the first inequality is confirmed since $1-d_{i j} \leq 1-c_{i j}$ or equivalently, $0 \leq c_{i j} \leq d_{i j} \leq 1$,

447 and the second inequality is derived because $\tilde{w}_{i j}^{U U}$ is an optimal solution of (4.6) and $\tilde{w}_{i j}^{L U}$

448 is a feasible solution of this maximization problem.

449 Furthermore, since $b_{i j}+d_{i j} \leq 1$, or equivalently, $b_{i j} \leq 1-d_{i j}$, as per (4.4), we have 450

$$
\tilde{z}_{i}^{U L} \triangleq \sum_{j=1}^{m} b_{i j} \tilde{w}_{i j}^{U L} \leq \sum_{j=1}^{m}\left(1-d_{i j}\right) \tilde{w}_{i j}^{U L} \leq \sum_{j=1}^{m}\left(1-d_{i j}\right) \tilde{w}_{i j}^{L U} \triangleq \tilde{z}_{i}^{L U}
$$


451 Once again, the first inequality holds as $b_{i j} \leq 1-d_{i j}$, and the second inequality comes

452 from the fact that $\tilde{w}_{i j}^{L U}$ is an optimal solution of the maximization problem in (4.4) and

$453 \tilde{w}_{i j}^{U L}$ is a feasible solution. The proof is thus completed. Q.E.D.

454 Theorem 4.1 indicates that the optimal aggregated value of $x_{i} \in X$ can be 455 characterized by a pair of intervals: $\left[\tilde{z}_{i}^{L L}, \tilde{z}_{i}^{U L}\right]$ and $\left[\tilde{z}_{i}^{L U}, \tilde{z}_{i}^{U U}\right]$. As $\tilde{z}_{i}^{L L} \leq \tilde{z}_{i}^{U L}, \tilde{z}_{i}^{L U} \leq \tilde{z}_{i}^{U U}$, 456 one can have $\tilde{z}_{i}^{L L} \leq \tilde{z}_{i}^{U L}, 1-\tilde{z}_{i}^{U U} \leq 1-\tilde{z}_{i}^{L U}$. Furthermore, since $\tilde{z}_{i}^{U L} \leq \tilde{z}_{i}^{L U}$, it is implied that $457 \tilde{z}_{i}^{U L}+1-\tilde{z}_{i}^{L U} \leq 1$. Therefore, written in an IVIFN format, the optimal aggregated value of 458 the alternative $x_{i} \in X$ can be given as

$$
\begin{aligned}
& \tilde{\alpha}_{i}=\left(\left[\tilde{z}_{i}^{L L}, \tilde{z}_{i}^{U L}\right],\left[1-\tilde{z}_{i}^{U U}, 1-\tilde{z}_{i}^{L U}\right]\right) \\
& =\left(\left[\sum_{j=1}^{m} a_{i j} \tilde{w}_{i j}^{L L}, \sum_{j=1}^{m} b_{i j} \tilde{w}_{i j}^{U L}\right],\left[\sum_{j=1}^{m} c_{i j} \tilde{w}_{i j}^{U U}, \sum_{j=1}^{m} d_{i j} \tilde{w}_{i j}^{L U}\right]\right)
\end{aligned}
$$

460 As the weight vectors $\tilde{W}_{i}^{L L}, \tilde{W}_{i}^{L U}, \tilde{W}_{i}^{U L}$, and $\tilde{W}_{i}^{U U}$ are independently determined by the 461 four linear programs (4.3), (4.4), (4.5) and (4.6), respectively, it is understandable that 462 they are generally different, i.e., $\tilde{W}_{i}^{L L} \neq \tilde{W}_{i}^{L U} \neq \tilde{W}_{i}^{U L} \neq \tilde{W}_{i}^{U U}$ for $x_{i} \in X$, or $\tilde{w}_{i j}^{L L} \neq \tilde{w}_{i j}^{L U}$ $463 \neq \tilde{w}_{i j}^{U L} \neq \tilde{w}_{i j}^{U U}$ for $i=1,2, \ldots, n$ and $j=1,2, \ldots, m$. Therefore, it is not fair to compare the 464 aggregated values of all alternatives based on the different weight vectors. A more 465 reasonable common ground for comparing the aggregated values is to derive a unified 466 weight vector and apply this same weight vector to all alternatives. The following 467 discussions aim to obtain such a weight vector. The general procedure is similar to that 468 reported in [20], but it has been adapted to accommodate the specific structure of IVIFNs. 469 Since $X$ is a non-inferior alternative set, no alternative dominates or is dominated by 470 any other alternative. Hence, when all alternatives, rather than a single alternative in (4.3) 471 and (4.4), have to be considered, the contribution to the objective function from each 472 alternative should be treated with an equal weight of $1 / n$. Therefore, parallel to (4.3) and 473 (4.4), we have the following two aggregated linear programs. 
474

$$
\min \left\{z_{0}^{L L}=\frac{\sum_{i=1}^{n} \sum_{j=1}^{m} a_{i j} w_{j}}{n}\right\}
$$

$$
\text { s.t. }\left\{\begin{array}{c}
w \in H, \\
\sum_{j=1}^{m} w_{j}=1
\end{array}\right.
$$

475 and

476

$$
\max \left\{z_{0}^{L U}=\frac{\sum_{i=1}^{n} \sum_{j=1}^{m}\left(1-d_{i j}\right) w_{j}}{n}\right\}
$$

$$
\text { s.t. }\left\{\begin{array}{c}
w \in H, \\
\sum_{j=1}^{m} w_{j}=1
\end{array}\right.
$$

477 Note that (4.9) can be converted to an equivalent maximization linear programming 478 model in (4.11) by multiplying its objective function with -1 .

479

$$
\max \left\{\bar{z}_{0}^{L L}=-\frac{\sum_{i=1}^{n} \sum_{j=1}^{m} a_{i j} w_{j}}{n}\right\}
$$

$$
\text { s.t. }\left\{\begin{array}{c}
w \in H, \\
\sum_{j=1}^{m} w_{j}=1
\end{array}\right.
$$

480 Since (4.10) and (4.11) are both maximization problems and share the same constraints, 481 if we treat the two objective functions as equally important, a typical way to translate the 482 bi-objective linear programs into a single linear program is given below: 


$$
\max \left\{z^{L}=\frac{\sum_{i=1}^{n} \sum_{j=1}^{m}\left(1-a_{i j}-d_{i j}\right) w_{j}}{n}\right\}
$$

$$
\text { s.t. }\left\{\begin{array}{c}
w \in H, \\
\sum_{j=1}^{m} w_{j}=1
\end{array}\right.
$$

484 By applying the same procedure, (4.5) and (4.6) can be transformed to the following 485 linear program:

486

$$
\max \left\{z^{U}=\frac{\sum_{i=1}^{n} \sum_{j=1}^{m}\left(1-b_{i j}-c_{i j}\right) w_{j}}{n}\right\}
$$

$$
\text { s.t. }\left\{\begin{array}{c}
w \in H, \\
\sum_{j=1}^{m} w_{j}=1
\end{array}\right.
$$

Once again, as (4.12) and (4.13) are both maximization problems and have the same 488

489 constraints, they can be combined to formulate the following linear program:

$$
\max \left\{z=\frac{\sum_{i=1}^{n} \sum_{j=1}^{m}\left(2-a_{i j}-b_{i j}-c_{i j}-d_{i j}\right) w_{j}}{n}\right\}
$$

$$
\text { s.t }\left\{\begin{array}{c}
w \in H, \\
\sum_{j=1}^{m} w_{j}=1
\end{array}\right.
$$

\section{$490 \quad$ Remark 4.1}

491 If $a_{i j}=b_{i j}$ and $c_{i j}=d_{i j}, i=1,2, \ldots, n ; j=1,2, \ldots, m$, the IVIFNs in the decision matrix are 492 reduced to IFNs, and (4.14) is equivalent to Eq. (15) in [20] if the weight constraint $493 w \in H$ in (4.14) takes the same form of being bounded on the lower and upper sides as 494 that in [20]. From this perspective, the proposed approach can be treated as a natural 495 extension of the work reported in [20] from the IFN to IVIFN environment. 
Similarly, the linear programming model (4.14) can be easily solved by using the 497 simplex method or an appropriate optimization computer package. Denote its optimal 498 solution by $w^{0}=\left(w_{1}^{0}, w_{2}^{0}, \cdots, w_{m}^{0}\right)^{T}$, and follow the similar notation as (4.7) to define:

499

$$
\begin{aligned}
& \tilde{z}_{i}^{0 L L} \triangleq \sum_{j=1}^{m} a_{i j} \tilde{w}_{j}^{0} \\
& \tilde{z}_{i}^{0 U L} \triangleq \sum_{j=1}^{m} b_{i j} \tilde{w}_{j}^{0} \\
& \tilde{z}_{i}^{0 L U} \triangleq \sum_{j=1}^{m}\left(1-d_{i j}\right) \tilde{w}_{j}^{0} \\
& \tilde{z}_{i}^{0 U U} \triangleq \sum_{j=1}^{m}\left(1-c_{i j}\right) \tilde{w}_{j}^{0}
\end{aligned}
$$

500

As $a_{i j} \leq b_{i j}, c_{i j} \leq d_{i j}$ and $b_{i j}+d_{i j} \leq 1$, it follows that $z_{i}^{0 L L} \leq z_{i}^{0 U L}, z_{i}^{0 L U} \leq z_{i}^{0 U U}$ and $501 z_{i}^{0 U L} \leq z_{i}^{0 L U}$. Therefore, the optimal aggregated value of alternative $x_{i}$ using a unified 502 weight vector $w^{0}$ can be determined by a pair of closed intervals, $\left[z_{i}^{0 L L}, z_{i}^{0 U L}\right]$ and $503\left[z_{i}^{0 L U}, z_{i}^{0 U U}\right]$. Equivalently, this aggregated value can be expressed as an IVIFN:

504

$$
\begin{aligned}
& \tilde{\alpha}_{i}^{0}=\left(\left[z_{i}^{0 L L}, z_{i}^{0 U L}\right],\left[1-z_{i}^{0 U U}, 1-z_{i}^{0 L U}\right]\right) \\
& =\left(\left[\sum_{j=1}^{m} a_{i j} w_{j}^{0}, \sum_{j=1}^{m} b_{i j} w_{j}^{0}\right],\left[1-\sum_{j=1}^{m}\left(1-c_{i j}\right) w_{j}^{0}, 1-\sum_{j=1}^{m}\left(1-d_{i j}\right) w_{j}^{0}\right]\right) \\
& =\left(\left[\sum_{j=1}^{m} a_{i j} w_{j}^{0}, \sum_{j=1}^{m} b_{i j} w_{j}^{0}\right],\left[\sum_{j=1}^{m} c_{i j} w_{j}^{0}, \sum_{j=1}^{m} d_{i j} w_{j}^{0}\right]\right)
\end{aligned}
$$

505 for each $i=1,2, \ldots, n$. Note that $1-\sum_{j=1}^{m}\left(1-c_{i j}\right) w_{j}^{0}=\sum_{j=1}^{m} c_{i j} w_{j}^{0}$ and $1-\sum_{j=1}^{m}\left(1-d_{i j}\right) w_{j}^{0}=$ $506 \sum_{j=1}^{m} d_{i j} w_{j}^{0}$ are due to the fact that $\sum_{j=1}^{m} w_{j}^{0}=1$. Now Theorem 4.2 can be established.

507 Theorem 4.2 For $x_{i} \in X, i=1,2, \ldots, n$, assume that IVIFNs $\tilde{\alpha}_{i}$ and $\tilde{\alpha}_{i}^{0}$ are defined by 508 (4.8) and (4.16), respectively, then

$$
\left[\tilde{z}_{i}^{L L}, \tilde{z}_{i}^{U L}\right] \leq\left[z_{i}^{0 L L}, z_{i}^{0 U L}\right] \leq\left[z_{i}^{0 L U}, z_{i}^{0 U U}\right] \leq\left[\tilde{z}_{i}^{L U}, \tilde{z}_{i}^{U U}\right]
$$

511 solution of (4.3), (4.4), (4.5) and (4.6) as these linear programs share the same constraints. 
512 Note that $\tilde{W}_{i}^{L L}=\left(\tilde{w}_{i 1}^{L L}, \tilde{w}_{i 2}^{L L}, \cdots, \tilde{w}_{i m}^{L L}\right)^{T}$ and $\tilde{W}_{i}^{L U}=\left(\tilde{w}_{i 1}^{L U}, \tilde{w}_{i 2}^{L U}, \cdots, \tilde{w}_{i m}^{L U}\right)^{T}$ are an optimal

513 solution of (4.3) and (4.4), respectively, and $a_{i j} \leq b_{i j}$ and $b_{i j}+d_{i j} \leq 1$, it follows that

$514 \quad \tilde{z}_{i}^{L L} \triangleq \sum_{j=1}^{m} a_{i j} \tilde{w}_{i j}^{L L} \leq \sum_{j=1}^{m} a_{i j} w_{j}^{0} \triangleq z_{i}^{0 L L} \leq \sum_{j=1}^{m} b_{i j} w_{j}^{0} \leq \sum_{j=1}^{m}\left(1-d_{i j}\right) w_{j}^{0} \triangleq z_{i}^{0 L U} \leq \sum_{j=1}^{m}\left(1-d_{i j}\right) \tilde{w}_{i j}^{L U} \triangleq \tilde{z}_{i}^{L U}$

$515 \quad$ Here the first inequality holds true because $\tilde{w}_{i j}^{L L}$ is an optimal solution of (4.3) and $w_{j}^{0}$

516 is a feasible solution of this minimization problem. The $2^{\text {nd }}$ and $3^{\text {rd }}$ inequalities are due to

$517 a_{i j} \leq b_{i j} \leq 1-d_{i j}$. The last inequality is confirmed because the objective function value of a

518 feasible solution $w_{j}^{0}$ is always no more than that of an optimal solution $\tilde{w}_{i j}^{L U}$ for the

519 maximization problem (4.4). Therefore, we have $\tilde{z}_{i}^{L L} \leq z_{i}^{0 L L} \leq z_{i}^{0 L U} \leq \tilde{z}_{i}^{L U}$.

520 Similarly, as $\tilde{W}_{i}^{U L}=\left(\tilde{w}_{i 1}^{U L}, \tilde{w}_{i 2}^{U L}, \cdots, \tilde{w}_{i m}^{U L}\right)^{T}$ and $\tilde{W}_{i}^{U U}=\left(\tilde{w}_{i 1}^{U U}, \tilde{w}_{i 2}^{U U}, \cdots, \tilde{w}_{i m}^{U U}\right)^{T}$ are an

521 optimal solution of (4.5) and (4.6), respectively, and $c_{i j} \leq d_{i j}$ and $b_{i j}+d_{i j} \leq 1$, following

522 similar arguments, one can have

$523 \quad \tilde{z}_{i}^{U L} \triangleq \sum_{j=1}^{m} b_{i j} \tilde{w}_{i j}^{U L} \leq \sum_{j=1}^{m} b_{i j} w_{j}^{0} \triangleq z_{i}^{0 U L} \leq \sum_{j=1}^{m}\left(1-d_{i j}\right) w_{j}^{0} \leq \sum_{j=1}^{m}\left(1-c_{i j}\right) w_{j}^{0} \triangleq z_{i}^{0 U U} \leq \sum_{j=1}^{m}\left(1-c_{i j}\right) \tilde{w}_{i j}^{U U} \triangleq \tilde{z}_{i}^{U U}$

524 i.e., $\tilde{z}_{i}^{U L} \leq z_{i}^{O U L} \leq z_{i}^{O U U} \leq \tilde{z}_{i}^{U U}$.

$525 \quad$ By Definition 3.6, the proof of Theorem 4.2 is completed. Q.E.D.

526 Remark 4.2

527 Theorem 4.2 confirms that the aggregated value of $x_{i}$ obtained by (4.14) is always

528 bounded by that obtained by (4.3) - (4.6) in terms of Definition 3.6.

529 Based on the aforesaid analyses, we are now in a position to formulate an interval530 valued intuitionistic fuzzy approach to multiattribute decision making with incomplete 531 attribute weight information as described in the following steps.

532 Step 1. Obtain an optimal weight vector $w^{0}=\left(w_{1}^{0}, w_{2}^{0}, \cdots, w_{m}^{0}\right)^{T}$ as per (4.14).

533 Step 2. Determine the optimal aggregated value $\tilde{\alpha}_{i}^{0}$ for all alternatives $x_{i} \in X$, $534 \quad i=1,2, \cdots, n$ by plugging $w^{0}$ into (4.16).

535 Step 3. Calculate the values of the score function $S\left(\tilde{\alpha}_{i}^{0}\right)$, accuracy function $H\left(\tilde{\alpha}_{i}^{0}\right)$, 536 membership uncertainty index $T\left(\tilde{\alpha}_{i}^{0}\right)$, and hesitation uncertainty index $G\left(\tilde{\alpha}_{i}^{0}\right)$ for each 537 alternative in a sequential order, and rank all alternatives as per Definition 3.5 and/or 
538 choose the best alternative(s).

539 Remark 4.3

540 In an actual decision process, it is often unnecessary to calculate the values for all 541 four functions. For instance, if the purpose of the decision problem is to choose the best

542 alternative(s) and the sequential order in Definition 3.5 is followed to compute the 543 function values, whenever no tie is found for the best value of a function (largest for $544 S(\cdot)$ and $H(\cdot)$, but smallest for $T(\cdot)$ and $G(\cdot))$, the best choice is ascertained and it is not 545 necessary to calculate remaining function values in any lower hierarchy as detailed in 546 Definition 3.5. Even if the decision problem is to obtain a full ranking of all alternatives, 547 calculations may terminate before all four functions are entertained. For an example, see 548 Section 5.

549 Remark 4.4

550 From the modeling process, one can understand that the proposed framework here is 551 able to handle incomplete weight information characterized by a subset of linear 552 relationships given in Section 4.1. In addition, the aggregation process is achieved 553 through a series of optimization models that take the individual IVIFN assessments as 554 input, and the conversion from IVIFNs to real values is delayed until the last step when 555 different alternatives' aggregated IVIFN values are compared. This treatment avoids loss 556 of information due to conversions at early stages. Another advantage of this framework is 557 its novel comparison method that is able to distinguish any two different IVIFNs as 558 shown in Section 3. In terms of limitations of the proposed approach, an inherent 559 assumption of the aggregation process is that the attributes are independent and the 560 individual membership and nonmembership functions are linearly additive. If other forms 561 of information fusion schemes are required, this model would not be applicable. In 562 addition, the proposed approach requires that all individual assessment information must 563 be provided as IVIFNs in full and no missing data are allowed in the decision matrix. 564 Further research is necessary to expand this approach to accommodate these needs for 565 different fusion mechanisms and missing assessment data.

\section{An illustrative example}


This section adapts an investment decision problem in [12] to demonstrate how to apply the proposed approach. Although this example is provided in the context of selecting an optimal investment opportunity from a list of four choices in respect to four attributes against which each choice is assessed, it should be noted that, as suggested and

571 illustrated by Merigo and Gil-Lafuente [24] and $\mathrm{Xu}$ and Yager [38], the proposed 572 approach can be easily applied to a host of practical decision problems that involve 573 choosing an optimal alternative from a list of alternatives when multiple attributes must 574 be onsidered. For instance, selecting the best candidate to fill a tenure-track faculty 575 position at a Canadian university typically requires each recruitment committee member 576 to rank short-listed applicants based on different criteria such as research achievements/ 577 potentials, teaching/presentation skills, ability to attract funding from government 578 agencies and industries, and service to the profession and academic community. From 579 each committee member's perspective, this is a typical mutltiattribute decision making 580 situation and the weights among different attributes can be conveniently captured by a list 581 of constraints as shown in Section 4.1 and individual assessments may well be expressed 582 as IVIFNs.

583 For the following example, assume that a fund manager in a wealth management 584 firm is assessing four potential investment opportunities, $X=\left\{x_{1}, x_{2}, x_{3}, x_{4}\right\}$. The firm 585 mandates that the fund manager has to evaluate each investment against four attributes: 586 risk $\left(a_{1}\right)$, growth $\left(a_{2}\right)$, socio-political issues $\left(a_{3}\right)$, and environmental impacts $\left(a_{4}\right)$. In 587 addition, the fund manager is only comfortable with providing his/her assessment of each 588 alternative on each attribute as an IVIFN and the decision matrix is

$$
\tilde{R}=\left[\begin{array}{cccc}
([0.42,0.48],[0.4,0.5]) & ([0.6,0.7],[0.05,0.25]) & ([0.4,0.5],[0.2,0.5]) & ([0.55,0.75],[0.15,0.25]) \\
([0.4,0.5],[0.4,0.5]) & ([0.5,0.8],[0.1,0.2]) & ([0.3,0.6],[0.3,0.4]) & ([0.6,0.7],[0.1,0.3]) \\
([0.3,0.5],[0.4,0.5]) & ([0.1,0.3],[0.2,0.4]) & ([0.7,0.8],[0.1,0.2]) & ([0.5,0.7],[0.1,0.2]) \\
([0.2,0.4],[0.4,0.5]) & ([0.6,0.7],[0.2,0.3]) & ([0.5,0.6],[0.2,0.3]) & ([0.7,0.8],[0.1,0.2])
\end{array}\right]
$$

590 Each element of this matrix is an IVIFN, representing the fund manager's assessment 591 as to what degree an alternative is and is not an excellent investment as per an attribute. 592 For instance, the top-left cell, $([0.42,0.48]$, [0.4, 0.5]), reflects the fund manager's belief 593 that alternative $x_{1}$ is an excellent investment from a risk perspective $\left(a_{1}\right)$ with a margin 
594 of $42 \%$ to $48 \%$ and $x_{1}$ is not an excellent choice given its risk profile $\left(a_{1}\right)$ with a chance 595 between $40 \%$ and $50 \%$.

596 If the fund manager is able to provide the following attribute weight information: $597 w_{1}=0.13$ (risk), $w_{2}=0.17$ (growth), $w_{3}=0.39$ (socio-political issues), and $w_{4}=0.31$

598 (environmental impacts), calculations for our proposed approach start with Step 2 and 599 determine as follows the aggregated IVIFN values for the four alternatives by plugging 600 the given weights into (4.16): compute $S\left(\tilde{\alpha}_{3}\right)$ and $S\left(\tilde{\alpha}_{4}\right)$ as $x_{3}$ and $x_{4}$ are differentiated by the score function at a

611 higher priority level. Since the accuracy function values are also identical for $x_{1}$ and $x_{2}$,

612 it is necessary to move to the next priority level and calculate the membership uncertainty 613 index function values, $T\left(\tilde{\alpha}_{1}\right)=-0.0692, T\left(\tilde{\alpha}_{2}\right)=0.081$. Now a full ranking of the four 614 alternatives is obtained as: $x_{4} \succ x_{3} \succ x_{1} \succ x_{2}$.

615 This assumption of complete knowledge on attribute weights allows a comparative 616 study with other approaches in the current literature that require complete weight 617 information. The comparative study will utilize the decision matrix $\tilde{R}$ and the aforesaid 618 weights to compare the ranking result of our proposed approach with those obtained from 619 Procedure II ( $p=1)$ in Xu and Yager [38] and Xu [34] (both weighted arithmetic and 620 weighted geometric average aggregation operators).

621 To begin, the same decision matrix $\tilde{R}$ and weights are fed into the approach, 622 Procedure II, developed by Xu and Yager [38] (Note that Procedure I therein handles the 
623 case with IFN assessments rather than IVIFNs and, hence, is omitted here for the 624 comparative study). Let $p=1$ and $\tilde{R}$ be the resulting decision matrix from Step 1 therein. 625 Then, the closeness coefficient of each alternative (See Eq (73) on p258 in [38]) can be 626 rewritten as follows by using the notation in this article:

$$
c\left(x_{i}\right)=\frac{\sum_{j=1}^{m} w_{j}\left(2-\left(c_{i j}+d_{i j}\right)\right)}{\sum_{j=1}^{m} w_{j}\left(4-\left(a_{i j}+b_{i j}\right)-\left(c_{i j}+d_{i j}\right)\right)}
$$

628

Plugging the decision matrix and weights into (5.1) yields $c\left(x_{1}\right)=0.6125, c\left(x_{2}\right)=$ 0.6125, $c\left(x_{3}\right)=0.6433, c\left(x_{4}\right)=0.6517$. Based on the decision rule in $\mathrm{Xu}$ and Yager [38], 630 the larger a closeness coefficient of an alternative, the better the alternative. Therefore, 631 the ranking result from this approach is $x_{4} \succ x_{3} \succ\left\{x_{1}\right.$ ? $\left.x_{2}\right\}$, where the question mark 632 indicates that this approach cannot differentiate $x_{1}$ from $x_{2}$.

$633 \mathrm{Xu}$ [34] also develops weighted arithmetic and weighted geometric average 634 aggregation operators for IVIFN information fusions. Both operators are employed to 635 obtain rankings for the four alternatives here. As per the weighted arithmetic average 636 aggregation operator, the aggregated IVIFN value of an alternative is determined by [34, 637 Eqs. (14) and (16)]:

$$
\tilde{\alpha}_{i}=\sum_{j=1}^{m} \omega_{j} \tilde{r}_{i j}=\left(\left[1-\prod_{j=1}^{m}\left(1-a_{i j}\right)^{w_{j}}, 1-\prod_{j=1}^{m}\left(1-b_{i j}\right)^{w_{j}}\right],\left[\prod_{j=1}^{m} c_{i j}{ }^{w_{j}}, \prod_{j=1}^{m} d_{i j}{ }^{w_{j}}\right]\right)
$$

645 Section 3 here, one can determine that $s\left(\tilde{\alpha}_{1}\right)=0.30105, s\left(\tilde{\alpha}_{2}\right)=0.30105, s\left(\tilde{\alpha}_{3}\right)=0.4114$, $646 s\left(\tilde{\alpha}_{4}\right)=0.39005$. It is clear that the score function ranks the four alternatives as $647 x_{3} \succ x_{4} \succ\left\{x_{1} ? x_{2}\right\}$ and it cannot differentiate $x_{1}$ from $x_{2}$. Then, it is necessary to calculate 
648 the accuracy functions for the aggregated IVIFN values for $x_{1}$ and $x_{2}, H\left(\tilde{\alpha}_{1}\right)=0.81765$,

$649 H\left(\tilde{\alpha}_{2}\right)=0.81955$. Therefore, this approach generates a full ranking: $x_{3} \succ x_{4} \succ x_{2} \succ x_{1}$.

650 Similarly, the weighted geometric average aggregation operator given in Eqs. (15) 651 and (17) by Xu [34] is reproduced below for self-containment.

$$
\tilde{\alpha}_{i}=\prod_{j=1}^{m} \tilde{r}_{i j}{ }^{w_{j}}=\left(\left[\prod_{j=1}^{m} a_{i j}{ }^{w_{j}}, \prod_{j=1}^{m} b_{i j}{ }^{w_{j}}\right],\left[1-\prod_{j=1}^{m}\left(1-c_{i j}\right)^{w_{j}}, 1-\prod_{j=1}^{m}\left(1-d_{i j}\right)^{w_{j}}\right]\right)
$$

Plugging $\tilde{R}$ and the weights into (5.3) yields the following aggregated IVIFNs:

The corresponding score function values are $s\left(\tilde{\alpha}_{1}\right)=0.24455, s\left(\tilde{\alpha}_{2}\right)=0.24295$, $s\left(\tilde{\alpha}_{3}\right)=0.2852, s\left(\tilde{\alpha}_{4}\right)=0.32235$, resulting in a full ranking $x_{4} \succ x_{3} \succ x_{1} \succ x_{2}$.

$$
\begin{gathered}
\tilde{\alpha}_{1}=([0.4760,0.5972],[0.1915,0.3926]), \\
\tilde{\alpha}_{2}=([0.4211,0.6454],[0.2260,0.3546]), \\
\tilde{\alpha}_{3}=([0.4057,0.6112],[0.1632,0.2833]), \\
\tilde{\alpha}_{4}=([0.5081,0.6389],[0.2007,0.3016]) .
\end{gathered}
$$

In summary, the results of this comparison study can be shown in Table 1.

Table 1. A comparative study when attribute weight information is complete

\begin{tabular}{lll}
\hline Decision approach & Reference & Ranking result \\
\hline Procedure II, $p=1$ & Xu and Yager [38] & $x_{4} \succ x_{3} \succ\left\{x_{1} ? x_{2}\right\}$ \\
Arithmetic operator & $\mathrm{Xu}$ [34] & $x_{3} \succ x_{4} \succ x_{2} \succ x_{1}$ \\
Geometric operator & $\mathrm{Xu}$ [34] & $x_{4} \succ x_{3} \succ x_{1} \succ x_{2}$ \\
This approach & This article & $x_{4} \succ x_{3} \succ x_{1} \succ x_{2}$
\end{tabular}

Table 1 demonstrates the overall consistency of the ranking results based on the 663 proposed approach in this article and other approaches. All of the four approaches rank $664 x_{3}$ and $x_{4}$ as the first two alternatives, with $x_{4}$ being identified as the most preferred 665 investment opportunity by three approaches except the weighted arithmetic average 666 aggregation operator in $\mathrm{Xu}$ [34]. For the remaining two investment opportunities, $x_{1}$ and $667 x_{2}$, the weighted geometric average aggregation operator in $\mathrm{Xu}$ [34] and our approach 668 rank $x_{1}$ first, but the weighted arithmetic average aggregation operator in $\mathrm{Xu}$ [34] ranks $669 x_{2}$ in front of $x_{1}$, and the $\mathrm{Xu}$ and Yager [38] approach cannot distinguish these two 
670 alternatives. The subtle differences in ranking are simply due to the distinct information 671 fusion mechanisms in these approaches.

672 It should be noted that the two approaches in Xu [34] do not always provide a full 673 ranking for the alternatives under consideration because the comparison mechanism there 674 utilizes only score and accuracy functions. As indicated in Section 3, it is possible that 675 certain alternatives cannot be distinguished by these two functions only. Similarly, the 676 first approach in $\mathrm{Xu}$ and Yager [38] sometimes cannot differentiate all distinct 677 alternatives, either. Furthermore, to make the comparative study possible, it is assumed 678 that the attribute weight information is completely known as the other three approaches 679 cannot handle the case when attribute weights are incomplete.

680 In reality, however, complete weight information is not always readily available. 681 Instead only partial knowledge of attribute weights may be obtained as a group of linear 682 constraints such as those given in Section 4.1. For instance, assume that the fund manager 683 can only provide his/her incomplete knowledge about the weights as follows:

$$
\begin{aligned}
H= & \left\{0.15 \leq w_{1} \leq 0.3,0.15 \leq w_{2} \leq 0.25,\right. \\
& \left.0.25 \leq w_{3} \leq 0.4,0.3 \leq w_{4} \leq 0.45,2.5 w_{1} \leq w_{3}\right\}
\end{aligned}
$$

685 In this case, the other three approaches in the previous comparative study would not 686 be applicable, but the proposed approach in this article will be able to solve the problem. 687 According to (4.14), the following linear program is established.

688

$$
\begin{aligned}
& \max \left\{z=\left(1.2 w_{1}+2 w_{2}+1.4 w_{3}+1.3 w_{4}\right) / 4\right\} \\
& \text { s.t. }\left\{\begin{array}{l}
0.15 \leq w_{1} \leq 0.3, \\
0.15 \leq w_{2} \leq 0.25, \\
0.25 \leq w_{3} \leq 0.4, \\
0.3 \leq w_{4} \leq 0.45, \\
2.5 w_{1} \leq w_{3}, \\
\sum_{j=1}^{4} w_{j}=1
\end{array}\right.
\end{aligned}
$$

689 Solving this linear programming, one can obtain its optimal solution as:

$$
w^{0}=\left(w_{1}^{0}, w_{2}^{0}, w_{3}^{0}, w_{4}^{0}\right)^{T}=(0.1500,0.1750,0.3750,0.3000)^{T}
$$


Note that the derived weight vector slightly differs from that given in the comparative

692

693

694

695

696 study. Plugging the weight vector $w^{0}$ and individual assessments in the decision matrix $\tilde{R}$ into (4.16), the optimal aggregated values for the four alternatives are determined.

$$
\begin{gathered}
\tilde{\alpha}_{1}^{0}=([0.48300,0.60700],[0.18875,0.38125]), \\
\tilde{\alpha}_{2}^{0}=([0.44000,0.65000],[0.22000,0.35000]), \\
\tilde{\alpha}_{3}^{0}=([0.4750,0.6375],[0.1625,0.2800]), \\
\tilde{\alpha}_{4}^{0}=([0.5325,0.6475],[0.2000,0.3000]) .
\end{gathered}
$$

Next, the score function is calculated for each aggregated value as

$$
S\left(\tilde{\alpha}_{1}^{0}\right)=0.2600, S\left(\tilde{\alpha}_{2}^{0}\right)=0.2600, S\left(\tilde{\alpha}_{3}^{0}\right)=0.3350, S\left(\tilde{\alpha}_{4}^{0}\right)=0.3400
$$

Obviously, $S\left(\tilde{\alpha}_{4}^{0}\right)>S\left(\tilde{\alpha}_{3}^{0}\right)>S\left(\tilde{\alpha}_{1}^{0}\right)=S\left(\tilde{\alpha}_{2}^{0}\right)$ and, hence, $x_{4} \succ x_{3} \succ\left\{x_{1}\right.$ ? $\left.x_{2}\right\}$. The score function values indicate that the most preferred alternative is $x_{4}$, followed by $x_{3}$, and then $x_{1}$ and $x_{2}$. As $\tilde{\alpha}_{1}^{0} \neq \tilde{\alpha}_{2}^{0}$, the question mark between $x_{1}$ and $x_{2}$ indicates that their ranking cannot be determined by the score function as both have the same score of 0.2600. If the purpose is to choose the best investment alternative only, the problem is completed now. On the other hand, if the fund manager is interested in a full ranking of the four investments, it is necessary to calculate the accuracy function values of $\tilde{\alpha}_{1}^{0}$ and $\tilde{\alpha}_{2}^{0}$ for the first two investment opportunities.

By Definition 3.2, it is easy to verify that

$$
H\left(\tilde{\alpha}_{1}^{0}\right)=H\left(\tilde{\alpha}_{2}^{0}\right)=0.8300
$$

Once again, the ranking between $x_{1}$ and $x_{2}$ still cannot be determined. Therefore, we proceed with the membership uncertainty index $T\left(\tilde{\alpha}_{i}^{0}\right)(i=1,2)$

$$
T\left(\tilde{\alpha}_{1}^{0}\right)=-0.0685, T\left(\tilde{\alpha}_{2}^{0}\right)=0.08
$$

As $S\left(\tilde{\alpha}_{1}^{0}\right)=S\left(\tilde{\alpha}_{2}^{0}\right), H\left(\tilde{\alpha}_{1}^{0}\right)=H\left(\tilde{\alpha}_{2}^{0}\right), T\left(\tilde{\alpha}_{1}^{0}\right)<T\left(\tilde{\alpha}_{2}^{0}\right)$, by Definition 3.5, we have $x_{1} \succ x_{2}$. Therefore, a full ranking of all four alternatives is obtained as

$$
x_{4} \succ x_{3} \succ x_{1} \succ x_{2}
$$

\section{Conclusions}


This article puts forward a framework to tackle multiattribute decision making 718 problems with interval-valued intuitionistic fuzzy assessments and incomplete attribute

719 weight information. The proposed approach employs a series of optimization models to 720 derive a unified weight vector and this weight vector is then applied to synthesize 721 individual IVIFN assessments into an aggregated IVIFN value for each alternative. To 722 rank alternatives based on aggregated IVIFNs, a novel method is devised to compare any 723 two IVIFNs.

724 An illustrative example is developed to demonstrate how to apply the proposed 725 procedure and comparative studies show its overall ranking consistency with existing 726 research. Numerical experiments illustrate the benefit of this proposed framework: it is 727 capable for handling incomplete weights and a full ranking can always be obtained as 728 long as the alternatives’ aggregated IVIFN values are not identical. On the other hand, 729 this approach is not without limitations as the decision matrix must be provided without 730 any missing assessments and the information fusion mechanism is essentially linearly 731 additive. Further research is required to extend the proposed approach to accommodate 732 the cases when the decision matrix contains missing data and different aggregation 733 schemes have to be entertained.

\section{REFERENCES}

735 [1] K. Atanassov, Intuitionistic fuzzy sets, Fuzzy Sets and Systems 20 (1986) 87-96.

736 [2] K. Atanassov, Operators over interval-valued intuitionistic fuzzy sets, Fuzzy Sets and $737 \quad$ Systems 64 (1994) 159-174.

738 [3] K. Atanassov, G. Gargov, Interval-valued intuitionistic fuzzy sets, Fuzzy Sets and 739 Systems 31 (1989) 343-349.

740 [4] K. Atanassov, G. Pasi, R.R. Yager, Intuitionistic fuzzy interpretations of multi-criteria 741 multiperson and multi-measurement tool decision making, International Journal of $742 \quad$ Systems Science 36 (2005) 859-868.

743 [5] H. Bustince, P. Burillo, Correlation of interval-valued intuitionistic fuzzy sets, Fuzzy $744 \quad$ Sets and Systems 74 (1995) 237-244.

745 [6] S.M. Chen, J.M. Tan, Handling multicriteria fuzzy decision-making problems based 746 on vague set theory, Fuzzy Sets and Systems 67 (1994) 163-172. 
[7] G. Deschrijver, Arithmetic operators in interval-valued fuzzy set theory, Information Sciences, 177 (2007), 2906-2924.

749 [8] G. Deschrijver, A representation of t-norms in interval-valued $L$-fuzzy set theory,

$750 \quad$ Fuzzy Sets and Systems 159 (2008) 1597-1618.

751 [9] G. Deschrijver, E.E. Kerre, On the position of intuitionistic fuzzy set theory in the

752 framework of theories modelling imprecision, Information Sciences 177 (2007) 1860

$753-1866$.

754 [10] W.L. Gau, D.J. Buehrer, Vague sets, IEEE Transactions on Systems, Man, and

755 Cybernetics 23 (1993) 610-614.

756 [11] J. Goguen, L-fuzzy sets, Journal of Mathematical Analysis and Applications 18 $757 \quad$ (1967) 145-174.

758 [12] F. Herrera, E. Herrera-Viedma, Linguistic decision analysis: steps for solving 759 decision problems under linguistic information, Fuzzy Sets and Systems 115 (2000) $760 \quad 67-82$.

761 [13] D.H. Hong, A note on correlation of interval-valued intuitionistic fuzzy sets, Fuzzy $762 \quad$ Sets and Systems 95 (1998) 113-117.

763 [14] D.H. Hong, C.H. Choi, Multicriteria fuzzy decision-making problems based on 764 vague set theory, Fuzzy Sets and Systems 114 (2000) 103-113.

[15] W.L. Hung, J.W. Wu, Correlation of intuitionistic fuzzy sets by centroid method, Information Sciences 144 (2002) 219 - 225.

[16] C.L. Hwang, K. Yoon, Multiple Attribute Decision Making: Methods and Applications, Springer, Berlin, Heideberg, New York, 1981.

[17] F. Karray, C.W. de Silva, Soft Computing and Intelligent Systems Design: Theory, Tools and Applications, Addison-Wesley, 2004.

[18] S.H. Kim, S.H. Choi, J.K. Kim, An interactive procedure for multiple attribute group decision making with incomplete information: range based approach, European Journal of Operational Research 118 (1999) 139-152.

[19] D.F. Li, An approach to fuzzy multiattribute decision making under uncertainty, Information Sciences 169 (2005) 97-112.

[20] D.F. Li, Multiattribute decision making models and methods using intuitionistic fuzzy sets, Journal of Computer and System Sciences 70 (2005) 73-85. 
[21] D.F. Li, J.B. Yang, Fuzzy linear programming technique for multiattribute group decision making in fuzzy environments, Information Sciences 158 (2004) 263-275.

[22] H.W. Liu, G.J. Wang, Multi-criteria decision-making methods based on intuitionistic fuzzy sets, European Journal of Operational Research 179 (2007) 220-233.

[23] X.D. Liu, S.H. Zheng, F.L. Xiong, Entropy and subsethood for general intervalvalued intuitionistic fuzzy sets, Lecture Notes in Artificial Intelligence vol. 3613, 2005, pp.42-52.

[24] J.M. Merigo and A.M. Gil-Lafuente, The induced generalized OWA operator, Information Sciences, 179 (2009) 729-741.

[25] T.K. Mondal, S.K. Samanta, Topology of interval-valued intuitionistic fuzzy sets, Fuzzy Sets and Systems 119 (2001) 483-494.

[26] K.S. Park, Mathematical programming models for charactering dominance and potential optimality when multi-criteria alternative values and weights are simultaneously incomplete. IEEE Transactions on Systems, Man, and Cybernetics, Part A: Systems and Humans 34 (2004) 601-614.

[27] E. Szmidt, J. Kacprzyk, Using intuitionistic fuzzy sets in group decision making, Control and Cybernetics 31 (2002) 1037-1053.

[28] E. Szmidt, J. Kacprzyk, A consensus-reaching process under intuitionistic fuzzy preference relations, International Journal of Intelligent Systems 18 (2003) 837-852.

[29] W. Wang, Z. Wang, An approach to multi-attribute interval-valued intuitionistic fuzzy decision making with incomplete weight information, In Proceedings of Fifth International Conference on Fuzzy Systems and Knowledge Discovery, pp. 346-350, Jinan, China, October 2008.

[30] Z. Wang, W. Wang, K.W. Li, Multi-attribute decision making models and methods under interval-valued intuitionistic fuzzy environment, In Proceedings of the 2008 Chinese Control and Decision Conference, pp. 2336-2341, Yantai, China, July 2008.

[31] Z. Xu, On correlation measures of intuitionistic fuzzy sets, Lecture Notes in Computer Science, vol. 4224, 2006, pp.16-24 [The 7th International Conference on Intelligent Data Engineering and Automated Learning, Burgos, Spain].

[32] Z. Xu, Intuitionistic fuzzy aggregation operators, IEEE Transactions on Fuzzy Systems 15 (2007) 1179-1187. 
809 [33] Z. Xu, Intuitionistic preference relations and their application in group decision 810 making, Information Sciences 177 (2007) 2363-2379.

811 [34] Z. Xu, Methods for aggregating interval-valued intuitionistic fuzzy information and 812 their application to decision making, Control and Decision 22 (2007) 215-219 (in 813 Chinese).

814 [35] Z. Xu, Multiple-attribute group decision making with different formats of preference 815 information on attributes, IEEE Transactions on Systems, Man, and Cybernetics, Part 816 B: Cybernetics 37 (2007) 1500-1511.

817 [36] Z. Xu, J. Chen, An interactive method for fuzzy multiple attribute group decision 818 making, Information Sciences 177 (2007) 248-263.

819 [37] Z. Xu, R.R. Yager, Some geometric aggregation operators based on intuitionistic 820 fuzzy sets, International Journal of General Systems 35 (2006) 417-433.

821 [38] Z. Xu, R.R. Yager, Dynamic intuitionistic fuzzy multi-attribute making, 822 International Journal of Approximate Reasoning 48 (2008), 246-262.

823 [39] L.A. Zadeh, Fuzzy sets, Inform. Control, 8 (1965) 338-353.

824 [40] L.A. Zadeh, Toward a generalized theory of uncertainty (GTU) - an outline, 825 Information Sciences, 172 (2005), 1-40.

826 [41] L.A. Zadeh, Is there a need for fuzzy logic? Information Sciences, 178 (2008), 2751$827 \quad 2779$. 